\title{
Priorities for the development and evaluation of support after suicide bereavement in the UK: results of a discussion group
}

Alexandra Pitman MRCPsych PhD

Honorary Research Associate,

UCL Division of Psychiatry; Camden and Islington NHS Foundation Trust

Adelia Khrisna Putri BA

MSc student

UCL Division of Psychiatry

Nina Kennedy BA DLIS

Chair, Suicide Bereaved Network

Tanisha De Souza BSc

MSc student

UCL Division of Psychiatry

Michael King FRCPsych FRCP FRCGP PhD

Professor of Primary Care Psychiatry

UCL Division of Psychiatry; Camden and Islington NHS Foundation Trust

David Osborn MRCPsych PhD

Professor of Psychiatric Epidemiology

UCL Division of Psychiatry; Camden and Islington NHS Foundation Trust

\begin{abstract}
We present the results of a group discussion conducted to identify research priorities among people bereaved by suicide regarding support needs. The authors' research team had recently published evidence from a UK-wide sample of young bereaved adults showing that those bereaved by suicide had a greater probability of suicide attempt than those bereaved by other cause of sudden death. For this study, ten UK-based adults bereaved by the suicide of a partner or family member were invited to join a group discussion to identify and prioritise an intervention to evaluate. These priorities were discussed in the context of unmet needs for support, identifying a need to develop and evaluate: immediate outreach after suicide; diversification and development of peer support services; and individual psychological support for those who feel suicidal. The group also suggested five key outcome measures: isolation; stigma; psychological health; day-to-day social functioning; and functioning in a work or caregiver role. The views presented in this discussion are a valuable contribution to the design of research that will inform national public health policy and the suicide prevention strategy for England.
\end{abstract}


Keywords: Suicide, support services, peer support, postvention, suicide prevention, outcome measures

\section{Introduction}

Providing support for people bereaved by suicide is a key objective of the suicide prevention strategy for England (Department of Heath, 2012) and is also a key international public health priority (World Health Organisation, 2014). The umbrella term 'postvention' describes any support provided to family, friends, colleagues and health professionals after someone dies by suicide, with the aim of enabling recovery and preventing further suicides from happening (Andriessen, 2009). Our recent systematic review of all published studies describing the impact of sudden bereavement critiqued and synthesised the findings of 57 international research studies, and provided clear evidence that people bereaved by suicide are vulnerable to depression, psychiatric admission, and suicide when compared with people bereaved by other causes of death (Pitman \& Osborn et al, 2014). Our British survey of young bereaved adults also found a greater probability of suicide attempt and poor occupational functioning in people bereaved by suicide (whether related to the deceased or not) compared with those bereaved by other causes of sudden death (Pitman \& Osborn et al, 2016a). We also found significantly higher levels of perceived stigma, shame, responsibility and guilt in people bereaved by suicide (Pitman \& Osborn et al, 2016b).

Despite the clear vulnerabilities of people bereaved by suicide, and this high level of policy attention (World Health Organisation, 2014) few tailored interventions for this group have been evaluated in a randomised trial (McDaid, Trowman \& Golder et al, 2008). The few published trials demonstrate weak evidence for effectiveness, and none were UK-based (McDaid, Trowman \& Golder et al, 2008). This means that although a network of support after suicide (see Figure 1) is currently growing in the UK (Public Health England, 2015), it is not yet evidence based. The role of the NHS in this support network is also not clear, with the suicide prevention strategy for England suggesting only that general practitioners (GPs) should be 'vigilant to the potential vulnerability of family members' after a suicide (Department of Heath, 2012). An exception is after suicides of patients recently under the care of psychiatric services, in which cases NHS guidelines recommend that mental health teams should offer families and carers 'appropriate and effective support' (National Patient Safety Agency, 2009). In practice, the majority of support available is provided by the voluntary sector (Public Health England, 2015).

Figure 1: Bereavement Support Triangle (with permission of Di Stubbs, Support after Suicide Partnership)

Our research team perceived a clear need to develop and evaluate an intervention to improve the health and social functioning of people bereaved by suicide, contributing to the implementation of an evidence-based suicide prevention strategy. We had already collected on-line and interview data from a national sample of bereaved adults in order to identify positive and negative experiences of support after suicide, and were aware of other published 
surveys on this topic conducted outside the UK (Dyregrov, 2011). This work had given us a sense of the broad range of formal and informal support that people valued after bereavement by suicide. However, we wished to involve the public more actively in directing the next stage of our research by identifying and prioritising an intervention to evaluate. We took advice from the UK's NIHR Research Design Service on the ways in which we could increase patient and public involvement (PPI) in this research programme. This report describes a group discussion we conducted to guide our choice of intervention, as a means of inviting views on priorities.

Our objective was to conduct a group discussion to elicit the views of UK-based people bereaved by suicide on priorities for developing and evaluating support services delivered after a suicide. To improve our understanding of the context we also aimed to elicit the group's views on unmet needs for support, specific support for those who feel suicidal, and the appropriate role of GPs, as well as meaningful outcome measures to use in a trial. Our second objective was to publish these views as a report, both to provide a research resource and to document perceived unmet needs for support. Previous surveys of the needs of people bereaved by suicide had been conducted outside the UK (Dyregrov, 2011), but we wished to represent the perceived unmet needs for support of UK-based people bereaved by the suicide of a relative or non-relative. Rather than conducting a formal qualitative analysis of data, we aimed to present a summary account of the discussion, verifying this with participants collaboratively.

\section{Method}

We recruited participants bereaved by suicide by emailing contacts at a range of voluntary sector organisations (Coroners' Court Support Service; Suicide Bereaved Network; Survivors of Bereavement by Suicide; Cruse Bereavement Care; Child Bereavement UK, Widowed by Suicide, the WAY Foundation) and by contacting local GPs in Camden and Islington (two boroughs in central north London). We invited participation from people who had been bereaved by the suicide of any close contact, with no age restrictions or limits on the period elapsed since the loss. Our geographical scope was limited to people who were based in the South East of England due to travel practicalities.

The invitation explained that the purpose of the group discussion was 'to find out what the priorities of bereaved people are in terms of setting up and evaluating services after a suicide'. Invitees were emailed an information sheet, including details of anonymity and confidentiality, and a consent form, which explained that the views expressed would be published as a report. This consent form also collected information on basic demographics and a subjective rating of experiences of support after bereavement, ranked using a visual analogue scale from 0 (very negative) to 10 (very positive). From available volunteers we used maximum variation sampling to select up to ten participants, reflecting a range of kinships, age groups, time elapsed since the bereavement, and experiences of support after bereavement. 
The 10 participants ( 2 men and 8 women) ranged in age from 47 to 73 (mean age=63.1; median=64), and had travelled from counties across the South East of England: Greater London, Surrey, Suffolk, Sussex, and Hertfordshire. The length of time that had elapsed since their loss ranged from 2 to 67 years (mean=20.5; median=15.5). Participants had been bereaved by the suicide of a son, daughter, parent, step-parent, sibling, spouse or partner. Two participants had been bereaved by the suicide of two relatives, and one had been bereaved by the suicide of three relatives (Further details of participants will not be provided out of respect for their anonymity and in keeping with prior confidentiality agreements). Ratings of their experiences of support after bereavement ranged from 1 (very negative) to 9 (very positive) with a median of 4 . All members of the discussion group had received help from peer support groups and over half had taken a facilitator role in those groups.

We chose an accessible venue at University College London in Central London, holding the meeting in March 2016. To maximise participation we timed the group to permit travel from locations outside London and within the school day. We were granted funding by the NIHR Research Design Service for participants' time and travel costs. All participants had the opportunity to ask questions about the study beforehand by telephone or email. On arriving at the venue group members had an opportunity to discuss the purpose and ground rules for the discussion group. It was explained that once a research priority had been identified, members would be invited to join the project's advisory group to advise on a study's future design.

The group was co-facilitated by a research psychiatrist, clinically experienced in managing distress after sudden bereavement (AP), and two MSc students from the UCL Division of Psychiatry (AP and TDS). The discussion lasted one hour and was audio recorded, with cofacilitators taking notes. Discussion was focussed by asking participants to consider four key questions, which set the context for the identification of research priorities:

- What services are missing from current provision of support after suicide in the UK?

- What is the role of GPs?

- What support would be appropriate for bereaved people who feel suicidal?

- When conducting research trials of interventions offered after suicide bereavement, what are the most meaningful measures of impact?

At the end of the discussion one co-facilitator summarised the key points raised, to check this was a fair account, explaining that a summary would be circulated for comments. There was an opportunity to ask further questions immediately afterwards. Based on the audio-recording of the discussion, the lead author summarised the topics discussed. This draft was emailed to participants, incorporating their comments into successive versions. This exercise was explicitly not a formal qualitative analysis, but the documentation and synthesis of a range of viewpoints. 


\section{Participants' views}

Throughout the course of the discussion, members of the group built up a picture of the disparity between the current provision of support and what they believed was necessary and appropriate as nationally. This is represented schematically in Figure 2, and provides the context for the views expressed under the four key headings below.

Figure 2: Schematic diagram of expressed need versus support available

\section{Gaps in service provision}

The overarching gap in service provision was seen to be the wide geographic variability in the support provided after suicide, spanning police and others who are first on the scene, coroners and other professionals, peer support groups, and individual psychosocial support. A need was expressed for geographical homogeneity to a set standard to prevent the current effect of 'a postcode lottery', arguing: 'There has to be a standard because they're vulnerable people'. There was a clear consensus that 'the best support is peer support' and that the majority of support provided after suicide in the UK is offered by the voluntary sector. It was acknowledged that whilst a network of peer support groups was growing across the UK, more groups were needed, both in frequency and across all localities. It was acknowledged that this relied on volunteers who were prepared to set up a group. The observation was made that whilst the majority of peer support was available as groups, voluntary sector organisations needed to diversify the support on offer, for example by providing home visits, immediate outreach, email and telephone support, or access to a private message board.

It was generally agreed that a major gap in current service provision was support offered in the immediate aftermath of the death; a period when the bereaved lack the resources to identify and access support. For those who felt unsupported by their social networks it felt important to have 'something to fill the silence from everyone around you'. The other rationale for providing some form of immediate support was the exclusion policy by many support services of people bereaved for less than three months. This was identified as a potentially vulnerable period for some individuals. Early support was described variously as a form of early intervention, immediate outreach, or immediate response system. This might be triggered or even provided by first responders such as Accident and Emergency staff, the police, or the GP. It was felt that all first responders should be able to provide immediate written information on sources of support, such as a copy of Help is at Hand (Public Health England, 2015) (for national organisations), or the contact details of a local Cruse Bereavement Care service or support group (eg. Survivors of Bereavement by Suicide, The Compassionate Friends).

The police force was identified as the most appropriate agency to provide immediate outreach because officers were perceived to attend the scene of the majority of suicides. However wide geographic variation was described in relation to the police's competence in this role, and there was a perceived need to train police officers in providing a compassionate response after a suicide in a way that did not further stigmatise the death. The group also 
acknowledged the potential conflict in the police's duty to investigate a suspicious death whilst also responding appropriately to a bereaved family. The majority view was that unless sufficient funding was available to provide ongoing training to police officers, it would be better for attending police officers to trigger a referral to another individual, who could then take on the outreach role.

One of the key responsibilities of an individual providing immediate outreach after suicide was judged to include providing information on local and national sources of support, but being clear that any of these sources could be accessed at any stage. This recognised that some people might not feel ready for groups at an early stage, preferring indirect contact such as a telephone helpline, email helpline, or messaging via an online forum. A tactfully-worded leaflet describing local services was thought to be a useful complement to the national services described in Help is At Hand (Public Health England, 2015). Being able to provide, or direct individuals to, individual or group psychological support was also felt to constitute an important function. Group members agreed that 'recognising suicide as a trauma is very important' for anyone bereaved by suicide, particularly those who had discovered the body of their loved one, as a means of preventing post-traumatic stress disorder. Some form of individual trauma-based counselling was felt to be appropriate (whether in the early stages or later on), but needed to be offered in a way that made it clear it was an option only if needed.

Group members thought it was essential to ensure that all members of a family received tailored support, appropriate to their age, with the needs of children particularly in mind. Children were thought to be relatively neglected in this context, and generally not able to access support by themselves. Child-centred support was suggested as well as indirect support for the adults responsible for their care. Another marginalised group identified was those not living in the area where the suicide had occurred. Local systems of immediate outreach would not normally be able to support such individuals, and alternative sources of outreach support were not apparent.

The ideal approach for an outreach worker to take was described as proactive whilst being sensitive to the potential for intrusiveness. Participants explained that they had needed someone to mobilise them to seek help, such was their inability to function at that point, commenting: 'you're not capable of reaching out - you need someone to come to you'. However they also recognised that others might find the intrusion too directive and overwhelming. There were concerns about the sensitivities to consider in naming the role of such an individual, particularly in contexts where the family did not want to consider or accept that the death had been suicide. In this case a job title such as Sudden Death Liaison Worker might be more acceptable, but this was to be balanced against the more 'honest' role description of Suicide Liaison Officer/Worker.

The importance of regular supervision for volunteer peer support workers was emphasised, both in terms of protecting their own emotional health as well as reducing variability across the service. Meeting in groups with a trained facilitator was regarded as the best means of providing an opportunity to ventilate, listen and gain training, although accessible telephone supervision was also suggested. Whilst recognising the funding implications, supervision of 
volunteers was regarded as worth this investment in terms of avoidance of burnout. Peer supervision was felt to represent a more economical option than professional supervision. One participant reported that Survivors of Bereavement by Suicide is working towards providing supervision for all its volunteers who lead groups.

The internet was discussed as a key means of identifying local sources of support, as there was a general sense of the newly-bereaved lacking access to a clear overview of service availability. For example, when a SOBS group in Suffolk surveyed its members over pathways into support it found that most members had found the group during internet searches, rather than being signposted to it directly. Although there was general awareness of the recently-launched Support After Suicide website, a hub for finding UK-based support (www.supportaftersuicide.org.uk/), it was felt that clearer guidance was needed over where to access individual versus group support. Whilst search engine optimisation meant that support organisations tended to be prioritised in searches of the term 'suicide', group members reported that it could sometimes be distressing to be confronted with sites promoting suicide methods. Older people were identified as a group who often lacked internet access, and might need better signposting to support groups, perhaps via GPs.

\section{Role of GPs}

Group members had very varying experiences of their GP's support, with only three having had positive experiences. GP contact had typically been initiated by the bereaved person rather than proactive outreach by the doctor. Generally it was felt that the GP's role was to offer medication and/or referrals for counselling where indicated. However being able to extend this role and signpost to local support groups would be greatly valued, particularly for those not internet-connected. One participant had appreciated it when a GP had 'stepped out of her GP role and become a helper'; suggesting sources of voluntary sector support. Being able to normalise the use of psychological interventions, and encourage uptake, were also seen as important because, as one participant observed 'I thought counselling was just for flaky people'. Another participant thought that her GP had dismissed her husband's suicide as 'just another bereavement', and felt fobbed off by the suggestion that she would feel better if she went for 'a good walk and had a cup of tea'. Given the variation in experiences, it was felt that GPs needed better training in how to respond to a person bereaved by suicide and a greater awareness of suicide's impact.

\section{Support for people who feel suicidal}

Feeling suicidal was acknowledged as a common experience after the suicide of a loved one. One participant reported that at a recent meeting of a peer support group 18 out of 20 members had raised their hands when asked if they felt suicidal. It was broadly agreed that asking such questions could be helpful as a means of normalising frightening feelings. In that setting 'it was a relief to know that they were not going crazy'. The only identifiable support for people who felt suicidal after the suicide of a loved was thought to be the telephone helplines provided by Samaritans, or PAPYRUS HopeLineUK for those under 35. One individual had sought out private therapy when feeling suicidal soon after the loss, and had found a trauma-focussed approach very helpful. 
Generally people felt that it was not always easy to identify those who might feel suicidal, but important to step up support for those who appeared to be experiencing prolonged or complicated grief. Those involved in leading peer support groups explained that they tended to identify people who seemed to be struggling, taking them aside after a meeting to provide details of organisations that might provide individual support, but without pressurising them to pursue this. Such services might include Cruse, Samaritans, or local mental health services. In Suffolk, local mental health services offered a self-referral wellbeing service (www.readytochange.org.uk/suffolk/pages/Home.aspx), providing group, individual, and online therapy for anyone in distress, regardless of whether or not they had a psychiatric diagnosis. However singling out people from groups to suggest they needed extra help was thought to be potentially stigmatising, with the recommendation that this needed sensitive handling. Group members felt that in some cases the worst affected individuals appeared to be in denial; focussing on giving others support whilst ignoring their own needs. This was identified as another rationale for providing supervision for peer group leaders.

\section{Meaningful measures of the impact of services}

Generally members acknowledged a need to measure outcomes to satisfy funders and to chart change. Some bereavement services apparently required clients to fill in tick-box forms at every weekly session, which to volunteer counsellors felt laborious but provided an objective means of showing improvements. The group did not object to the idea of asking bereaved individuals whether they felt suicidal. Indeed this was thought to be a good way of giving people permission to articulate such thoughts, providing relief by acknowledging that this sometimes happens after suicide bereavement. The group identified important psychological dimensions to measure in any trial: a reduced sense of isolation, depression, and stigma, and an increase in hope and confidence. Important improvements in functioning were listed as: being able to return to work or to a caregiver role, improved family dynamics, being able to sit in a group and talk freely, and even to move into a position where they wanted to facilitate a group and help others.

Being able to 'come to terms with what happened' and to 'become a real person again' was considered as important, although people agreed it was difficult to see how this could be captured in an objective measure. The interpersonal benefits of receiving support were also difficult to define, but included relief at having even one person to talk to; recognising that many people have no-one. To meet a peer bereaved by suicide was seen as an important means of redressing the sense of no-one understanding one's loss, and feeling less alone. To know that a service was available, regardless of whether one used it, was also regarded as comforting. Those who had taken part in peer support groups described instances of individuals who had sat silently crying throughout a group. This was regarded as a potentially positive experience if it provided that person with some comfort. Finally, the group agreed on five core outcomes to measure in a trial: isolation, stigma, psychological health, day-to-day social functioning, and functioning in a work or caregiver role. 


\section{Discussion}

This group discussion highlights the important role that people with lived experience of suicide bereavement play when working in partnership with researchers to identify an important and relevant research topic, and to advise on appropriate outcome measures. The group identified key priorities for researchers to address in terms of development and evaluation: immediate outreach after suicide, diversification and development of peer support services, and individual psychological support. They also identified five key health and social functioning outcomes to measure. Recording their views here provides a valuable resource for others working towards improving the health and social functioning of people bereaved by suicide. The ongoing involvement of members of this group as advisory group members also underlines their important role as co-researchers.

An added benefit of this exercise was the opportunity to record the perceived needs of a group of British people bereaved by the suicide of a sibling, partner, parent or child. Previous published surveys on this topic have not reflected the UK experience (Dyregrov, 2011), and this is important given cultural dimensions of grief. The views of our group broadly matched those in other high income countries; namely, immediate outreach after the death, and specific psychological work to address reactions to trauma (Dyregrov, 2011). Whilst in the US over two-thirds of people bereaved by suicide seek individual therapy following their loss (Mcmenamy, Jordan \& Mitchell, 2008), this was not the norm in our group or indeed in wider UK samples (unpublished data - in submission by first and senior authors). Another common unmet need described in the literature is for better support from family, friends, colleagues, and neighbours (Dyregrov, 2011); a topic covered only briefly in this group due to the focus on formal services. We have been able to feed back the perceived needs of this group to Public Health England, informing their development of guidance for local authorities in supporting people bereaved by suspected suicide (Public Health England, 2017).

It is important to note that that the primary aim of our group discussion was to advise on research priorities, and that their views on this and on perceived needs more generally may not be representative of all those in the UK bereaved by suicide. As a consequence of our recruitment method all members had participated in peer support groups, and a number had facilitated groups. In their own words they were therefore a 'self-selecting group' with regard to their readiness to seek as well as to provide help, and their views may not accord with those who had never sought help. Whilst the timing of the group was intended to promote participation of those with carer responsibilities, this approach did hamper participation of those in work. The age structure of the group meant that views were not representative of young adults, but our separate survey of young bereaved adults across the UK (Pitman \& Osborn et al, 2016a) has provided us with qualitative data on their needs for support.

Progress has already been made to address three of the service needs identified by this group. Firstly, the group identified a need for national standards on the provision of post-suicide support, including a national framework for immediate outreach after suicide. This is being addressed through the guidance recently produced by Public Health England (Public Health 
England, 2017). Secondly, the group identified a need to train GPs in how to respond to patients bereaved by suicide, including better awareness of the mental and physical impact of suicide (Pitman \& Osborn et al, 2014; Pitman \& Osborn et al, 2016a). This view matches the findings of formal qualitative research with people in Ireland bereaved by suicide, in which GPs are identified as ideally positioned to respond to their needs (Fhailí, Flynn \& Dowling, 2016). Qualitative work with GPs in England reveals their low confidence in knowing how to approach or help patients bereaved by a child's suicide (Foggin, McDonnell et al, 2016). To meet this gap the University of Manchester has developed training for health professionals on how to respond appropriately to parents bereaved by suicide. Remaining areas requiring research and policy attention include: the provision of supervision for those who provide peer support, the development of tailored psychological interventions for those who struggle to cope after a suicide, marketing of national services to those without internet access, and targeting bereaved people who do not live in the area where the suicide occurred.

The monitoring and evaluation of new services for people bereaved by suicide is crucial as a means of describing what a service is delivering, and to whom, as well as measuring outcomes. Such outcomes need to be meaningful to both commissioners and service users, and the suggestions of this group are extremely useful in planning future evaluations, including randomised controlled trials, of interventions provided. The results of applied health research are required to determine the optimum design of complex interventions such as systems of immediate outreach and novel psychological interventions, particularly using qualitative methods. Ongoing collaborations with people with lived experience of suicide, including those who took part in this discussion, will help ensure that services meet the needs of all stakeholders.

\section{Summary}

This group discussion brought together the views of ten people residing in southern England who had been bereaved by the suicide of a partner or family member. It identified the following as priorities for researchers to develop and evaluate: immediate outreach after suicide, diversification and development of peer support services, and individual psychological support for those who feel suicidal after a loved one's suicide. The group also suggested five key outcome measures: isolation, stigma, psychological health, day-to-day social functioning, and functioning in a work or caregiver role. This advice is currently being used to develop future research programmes for the development and evaluation of interventions to meet the needs of people bereaved by suicide. The participants in this discussion have an ongoing role in the design of a research programme arising from the discussion's findings, and their views have informed the development of Public Health England's guidance on the local provision of support after a suicide. As such, the views of this group have had immediate impact on public health policy and the suicide prevention strategy for England, and will continue to have impact through informing research design.

\section{Acknowledgements}


We would like to thank the ten bereaved people who participated in this discussion, the other individuals who volunteered to take part, and all those who have agreed ongoing involvement in this research programme. We are also grateful to the NIHR Research Design Service London Enabling Involvement Fund for financial support and advice on design, and to Noclor for NIHR Research Capability Funding to support Dr Alexandra Pitman. We thank Di Stubbs, Support After Suicide Partnership (www.supportaftersuicide.org.uk) for permission to reproduce Figure 1.

\section{References}

Andriessen K (2009). Can postvention be prevention? Crisis 30(1) 43-7.

Department of Health (2012). Preventing suicide in England: A cross-government outcomes strategy to save lives. Available from:

https://www.gov.uk/government/uploads/system/uploads/attachment_data/file/430720/Preven ting-Suicide-.pdf [accessed 19 October 2016].

Dyregrov K (2011). What do we know about needs for help after suicide in different parts of the world? Crisis 32(6) 310-8.

Fhailí MN, Flynn N, Dowling S (2016). Experiences of suicide bereavement: a qualitative study exploring the role of the GP. Br J Gen Pract 2016 66(643) e92-e98.

Foggin E, McDonnell S, Cordingley L et al (2016). GPs' experiences of dealing with parents bereaved by suicide: a qualitative study. Br J Gen Pract 66(651) e737.

McDaid C, Trowman R, Golder S, et al (2008). Interventions for people bereaved through suicide: systematic review. Br J Psychiatry 2008 Dec 1;193(6):438-43.

Mcmenamy JM, Jordan JR, Mitchell AM (2008). What do suicide survivors tell us they need? Results of a pilot study. Suicide Life Threat Behav 38(4) 375-89.

National Patient Safety Agency (2009). Preventing suicide: a toolkit for mental health services. Ref 1133. Available at: http://www.nrls.npsa.nhs.uk/resources/?entryid45=65297 [accessed 19 October 2016].

Pitman A, Osborn DPJ, King MB, Erlangsen A (2014). Effects of suicide bereavement on mental health and suicide risk. Lancet Psychiatry 1(1) 86-94.

Pitman AL, Osborn DPJ, Rantell K, King MB (2016a). Bereavement by suicide as a risk factor for suicide attempt: a cross-sectional national UK-wide study of 3432 young bereaved adults. BMJ Open 1 6(1).

Pitman AL, Osborn DPJ, Rantell K, King MB (2016b). The stigma perceived by people bereaved by suicide and other sudden deaths: A cross-sectional UK study of 3432 bereaved adults. J Psychosom Res 87 22-29. 
Public Health England, National Suicide Prevention Alliance (2015). Help is at hand: support after someone may have died by suicide. London: HMSO.

Public Health England (2017). Support after a suicide: a guide to providing local services. London: 2017. https://www.gov.uk/government/publications/support-after-a-suicide-a-guideto-providing-local-services

World Health Organisation (2014). Preventing suicide: a global imperative. Available at: http://www.who.int/mental_health/suicide-prevention/world_report_2014/en/ [accessed 19 October 2016]. 